\title{
Medium-term chest computed tomography (CT) follow-up of COVID-19 pneumonia patients after recovery to assess the rate of resolution and determine the potential predictors of persistent lung changes
}

\author{
Arshed Hussain Parry ${ }^{1 *} \mathbb{D}$, Abdul Haseeb Wani ${ }^{2}$, Naveed Nazir Shah ${ }^{3}$ and Majid Jehangir ${ }^{2}$
}

\begin{abstract}
Background: The data on medium-term follow-up of coronavirus disease-19 (COVID-19) pneumonia survivors is scarce. Medium-term follow-up will generate knowledge and help in devising a structured follow-up plan and to facilitate enrolment in clinical trials assessing the role of antifibrotic drugs in modifying the course of disease in order to avert long-term pulmonary sequelae of disease. The study was aimed to evaluate the lung findings on a medium-term follow-up (3 months or more) chest computed tomography (CT) in COVID-19 pneumonia survivors, assess the rate of resolution or persistence of lung abnormalities and to identify the initial demographic, clinical, and imaging characteristics that could potentially predict the persistence of lung abnormalities on follow-up.

Results: Out of the total study cohort of 81 patients, 46 (56.8\%) demonstrated complete resolution of lung findings and the remaining 35 (43.2\%) had residual lung opacities on follow-up CT. The most common type of residual abnormality was ground glass opacity (GGO) $(16 / 35 ; 45.7 \%)$, followed by parenchymal bands $(9 / 35 ; 25.7 \%)$, mixed pattern of GGO and parenchymal bands $(6 / 35 ; 17.2 \%)$, bronchiectasis $(6 / 35 ; 17.2 \%)$, and interlobular septal thickening (4/35; 11.4\%). Patients with residual abnormalities were older, had higher BMI, more comorbidities, lower SpO2, longer hospital stay, higher rate of intensive care unit (ICU) admission, higher WBC count, a higher CT severity score, and lower rate of steroid administration with all $p$ values $<0.05$.

Conclusion: Nearly half of post-COVID-19 survivors had residual lung abnormalities after $\geq 3$ months of follow-up. Certain clinico-radiological characteristics have the potential to identify the individuals at risk of having residual lung abnormalities on medium-term follow-up.
\end{abstract}

Keywords: COVID-19 survivors, Medium-term follow-up, Post-COVID-19 lung changes, Post-COVID-19 fibrosis, Persistent lung abnormalities, Pulmonary sequelae of COVID-19

\footnotetext{
* Correspondence: arshedparry@gmail.com

'Department of Radiodiagnosis, Sher-i-Kashmir Institute of Medical Sciences,

Srinagar, Jammu \& Kashmir, India

Full list of author information is available at the end of the article
}

\section{Springer Open}

( ) The Author(s). 2021 Open Access This article is licensed under a Creative Commons Attribution 4.0 International License, which permits use, sharing, adaptation, distribution and reproduction in any medium or format, as long as you give appropriate credit to the original author(s) and the source, provide a link to the Creative Commons licence, and indicate if changes were made. The images or other third party material in this article are included in the article's Creative Commons licence, unless indicated otherwise in a credit line to the material. If material is not included in the article's Creative Commons licence and your intended use is not permitted by statutory regulation or exceeds the permitted use, you will need to obtain permission directly from the copyright holder. To view a copy of this licence, visit http://creativecommons.org/licenses/by/4.0/. 


\section{Background}

Coronavirus disease 19 (COVID-19) has caused a major global health crisis. The disease which was declared as a pandemic by World Health Organization (WHO) on March 11, 2020 has infected an estimated 57.8 million till 21 November, 2020 and has claimed over 1.37 million lives across the world [1].

The diagnosis of COVID-19 hinges on the detection of viral nucleic acid in the respiratory secretions using reverse transcriptase polymerase chain reaction (RT-PCR). The consensus guidelines from various radiological societies across the world have discouraged the routine use of chest computed tomography $(\mathrm{CT})$ for establishing the diagnosis of COVID-19. However, performance of CT is essential in a subset of patients with severe disease and those showing respiratory deterioration during the course of illness. CT is also needed to monitor the course of disease or response to therapy [2-4].

The survivors of COVID-19 show varied clinical course after the initial recovery. Some patients recover fully with no residual symptoms or functional impairment; however, some experience persistent symptoms or functional impairment after the initial recovery [5].

As the long-term consequences of COVID-19 are not fully known at present, the data from previous coronavirus infections may provide useful insights as there may be important parallels between COVID-19 and its close "cousins" severe acute respiratory syndrome (SARS) and Middle East respiratory syndrome (MERS) which caused the outbreaks at 2003 and 2012, respectively.

In a longitudinal study of SARS survivors, $36 \%$ of patients had residual lung abnormalities at 3 months on chest X-ray (CXR) which decreased marginally to $30 \%$ at 6 months. When the radiographic abnormalities were correlated with pulmonary function tests, it was found that $16 \%$ of patients had impaired diffusion capacity of lungs (TLCo) at 6 months, thus implying that residual radiological abnormalities were physiologically relevant [6]. Similarly, in MERS survivors, $36 \%$ of patients had residual lung abnormalities in radiographs after a followup of 1 to 8 months [7].

The preliminary reports suggest that pulmonary parenchymal abnormalities do not completely resolve in all the survivors of COVID-19, and in some, the initial inflammation progresses into lung fibrosis [5]. A few published studies have assessed the temporal course of pulmonary findings in COVID-19 pneumonia, but they have mainly focused on the short-term changes in pulmonary opacities, usually up to 4 weeks [8-11].

Understanding the intermediate-term lung changes on CT may help in the identification of patients at risk of long-term COVID-19-induced lung compromise which would prompt initiation of appropriate treatment. It may also serve as a basis for selecting patients for future trials involving antifibrotic therapies.

The present study was aimed to determine the rate of complete radiological resolution on medium-term follow-up (3 months or more) and to identify the individuals who are at risk of developing long-term pulmonary sequelae.

\section{Methods}

\section{Study design and patient cohort}

This was a retrospective study conducted at a designated COVID-19 Care Centre in India. Institutional Ethical Committee (IEC) approval was obtained. The requirement for patient's informed consent was waived. RTPCR confirmed COVID-19 patients who had undergone chest CT during the initial or active phase of illness and at least one follow-up CT with a gap of at least 3 months between the two scans were enrolled in the study. If the patient had undergone more than one CT scan during the initial illness, then the CT with highest percentage of total lung involvement (highest CT severity score) was selected. The main indications for follow-up CT included persistent symptoms or functional impairment or persistent significant lung findings on follow-up chest $\mathrm{X}$ ray (CXR). In some cases, follow-up scans were performed on the relentless insistence of patients who were concerned about the sequelae of COVID-19.

The relevant demographic characteristics, clinical history, laboratory findings, pharmacological treatment received, and chest $\mathrm{CT}$ findings were collected and analyzed retrospectively.

\section{Data collection}

Demographic characteristics like age, gender, preexisting health conditions (hypertension, diabetes mellitus, chronic kidney disease (CKD), chronic liver disease (CLD), chronic obstructive pulmonary disease (COPD), malignancy, immunosuppression), and smoking history were documented. Clinical characteristics including main symptoms during initial illness and at the time of follow-up, percutaneous oxygen saturation (SpO2), and body measurements to calculate body mass index (BMI) were recorded. Laboratory indices including white blood cell count (WBC), neutrophil to lymphocyte ratio (NLR), C-reactive protein (CRP), and lactate dehydrogenase $(\mathrm{LDH})$ were collected. Important management and therapeutic data including outpatient vs inpatient, admission to intensive care unit (ICU), intubation, and the pharmacological treatment received were recorded.

\section{CT acquisition protocol and image interpretation}

All CT scans were performed on 16-Slice Siemens SOMATOM, Emotion Multidetector CT. Scans were acquired in a single breath-hold after setting up the patient 
in a head-first supine position in the CT gantry. The following scanning parameters were used: slice thickness $1-1.5 \mathrm{~mm}$, tube voltage $100-120 \mathrm{kVp}$, tube current of 90-130 mAs, and a beam pitch of 1.5. The tube current was regulated by an automatic exposure control system. Images were reconstructed using reconstruction increment of $0.7 \mathrm{~mm}$ into a slice thickness of $1 \mathrm{~mm}$. The images were viewed in lung window settings (width of 1200-1600 $\mathrm{HU}$ and centering of -500 to $-600 \mathrm{HU}$ ) and mediastinal window (width of 300-400 HU and centering of $40 \mathrm{HU}$ ).

Each patient had two sets of images (initial and followup). The CT scans were anonymized by replacing patient names with a code number by a CT technologist to make the readers blind to avoid possible bias. The CT images were independently analyzed by two experienced radiologists (P.A and W.A with 9 and 8 years of experience, respectively) blinded to the clinical data. In case of any disagreements between the two primary interpreting radiologists, a third radiologist (J.M, with more than 20 years of experience) adjudicated the final decision.

The following CT imaging characteristics were studied: (1) presence or absence of lung opacities; (2) distribution of lung opacities: unilateral vs bilateral lung involvement; (3) number of lobes affected; (5) dominant type of lung opacity: ground glass opacity (GGO), consolidation, mixed pattern of GGO, and consolidation and linear/curvilinear opacities.

Pulmonary opacities were categorized using the Fleischner Society glossary of terms for thoracic imaging [12]. To estimate the extent of lung involvement, a semi quantitative scoring system based on the visual calculation of percentage area of pulmonary opacification was used [13]. Each lung lobe was visually scored from 0 to 5 as follows: no involvement (score 0), less than $5 \%$ involvement (score 1), 5-25\% involvement (score 2), 26$50 \%$ involvement (score 3), 51-75\% involvement (score 4) and 76-100\% involvement (score 5). The scores of all five lobes were summed up to obtain a total CT severity score ranging from 0 (no involvement) to 25 (maximum involvement).

The interpretation of follow-up CT scans allowed categorization of patients into two groups based on the radiological clearance or persistence of pulmonary opacities: (1) patients with complete resolution of pulmonary opacities and (2) patients with residual pulmonary opacities.

\section{Statistical analysis}

Data were analyzed using the Statistical Package for the Social Sciences (SPSS Inc., Chicago, IL, version 21.0). Continuous variables were expressed as means and standard deviations. Categorical variables were expressed as counts and percentages. Fisher's exact test was used to examine the categorical variables. Two sample student $t$ test was used for comparison of continuous variables when the data was normally distributed, while Mann-Whitney $U$ test was used when the data was not normally distributed. A $p$ value less than 0.05 was considered statistically significant.

\section{Results}

The study included 81 patients comprising of 50 (61.7\%) males and 31 (38.3\%) females with a mean age of $51.8 \pm$ 11.7 years (range 32-69 years). Based on the disappearance or persistence of pulmonary opacities on follow-up $\mathrm{CT}$, patients were divided into two groups. A comparison of demographic, clinical, laboratory, therapeutic, and CT characteristics between the two groups is listed in Tables 1 and 2.

The average time interval between the initial and follow-up CT was 100.6 days (range 90-111 days). The follow-up CT revealed complete resolution of pulmonary opacities in 46/81 (56.8\%), whereas 35/81 (43.2\%) demonstrated residual pulmonary opacities (Fig. 1). The mean time lag between the initial and follow-up CT scans was not statistically different between the two follow-up groups of complete radiological resolution and persistent lung opacities ( $p$ value $=0.473$ )

Patients who demonstrated residual pulmonary opacities on follow-up CT were older (59.6 \pm 9.3 years) compared with the group demonstrating complete radiological resolution on follow-up CT $(45.8 \pm 13.8)(p$ value $=0.01858$ ).

Patients who showed persistent lung opacities on follow-up CT were more frequently overweight or obese $(21 / 35 ; 60 \%)$ compared with the group showing complete radiological resolution $(12 / 46 ; 26 \%)$ with a statistically significant difference $(p$ value $=0.003)$.

Though the group showing residual lung opacities had a higher number of smokers compared with the group showing complete radiological resolution (51 vs $28.3 \%$ ), the difference did not however reach statistical significance ( $p$ value $=0.65)$.

With regards to the presence or absence of underlying comorbid illness, a statistically higher percentage of patients demonstrating residual lung opacities had an underlying comorbidity compared with the resolved group $(80 \%$ vs $43.5 \%$; $p$ value $=0.0013)$.

No statistically significant difference was seen in the hospitalization rate between the resolved and residual groups (74 vs $88.6 \%$ ) ( $p$ value $=0.1583$ ). However, there was a significant difference in the rate of ICU admission between the groups of resolved and residual opacities $(6.5$ vs $28.6 \%)$ ( $p$ value $=0.0126)$.

The duration of hospital stay was significantly higher in the group demonstrating residual pulmonary opacities compared with the group demonstrating complete 
Table 1 Comparison of demographic, clinical, laboratory and therapeutic data between the two follow-up groups

\begin{tabular}{|c|c|c|c|}
\hline Parameter & $\begin{array}{l}\text { Complete radiological resolution } \\
\text { on follow-up CT }(n=46 ; 56.8 \%)\end{array}$ & $\begin{array}{l}\text { Residual lung findings } \\
\text { on follow-up CT ( } n=35 ; 43.2 \%)\end{array}$ & $p$ value \\
\hline Age (Mean $\pm S D)$ & $45.8 \pm 13.8$ & $59.6 \pm 9.3$ & 0.01858 \\
\hline Sex & $\begin{array}{l}M=29(63 \%) \\
F=17(37 \%)\end{array}$ & $\begin{array}{l}M=21(60 \%) \\
F=14(40 \%)\end{array}$ & 0.8205 \\
\hline \multicolumn{4}{|l|}{ BMl } \\
\hline $18-25$ & $34(74 \%)$ & $14(40 \%)$ & 0.003 \\
\hline$>25$ & $12(26 \%)$ & $21(60 \%)$ & \\
\hline \multicolumn{4}{|l|}{ Smoking history } \\
\hline Smoker & $13(28.3 \%)$ & $18(51.4 \%)$ & 0.6497 \\
\hline Non-smoker & $33(71.7 \%)$ & 17 (48.6\%) & \\
\hline \multicolumn{4}{|l|}{ Comorbidity } \\
\hline Absent & $26(56.5 \%)$ & $7(20 \%)$ & \\
\hline Present & $20(43.5 \%)$ & $28(80 \%)$ & \\
\hline Diabetes mellitus & 6 & 8 & \\
\hline Hypertension & 7 & 7 & \\
\hline COPD & 4 & 5 & 0.0013 \\
\hline CKD & 1 & 2 & \\
\hline CLD & 1 & 2 & \\
\hline CAD & 1 & 1 & \\
\hline Malignancy & 0 & 2 & \\
\hline Immune suppression & 0 & 1 & \\
\hline \multicolumn{4}{|l|}{ Hospitalization } \\
\hline Inpatient & $34(74 \%)$ & $31(88.6 \%)$ & 0.1583 \\
\hline Outpatient & $12(26 \%)$ & $4(11.4 \%)$ & \\
\hline In-ward hospitalization & $43(93.5 \%)$ & $25(71.4 \%)$ & 0.0126 \\
\hline ICU admission & $3(6.5 \%)$ & $10(28.6 \%)$ & \\
\hline \multicolumn{4}{|l|}{ Duration of hospitalization } \\
\hline (Mean days $\pm \mathrm{SD}$ ) & $7.6 \pm 2.3$ & $11.2 \pm 4.1$ & 0.000332 \\
\hline \multicolumn{4}{|c|}{ Initial percutaneous oxygen saturation (\%) } \\
\hline (Mean $\pm \mathrm{SD})$ & $92.3 \pm 3.8$ & $88.1 \pm 2.2$ & 0.00132 \\
\hline \multicolumn{4}{|l|}{ Initial WBC count } \\
\hline$\times 10^{3}($ Mean \pm SD $)$ & $5343 \pm 1456.4$ & $7987 \pm 2465.8$ & 0.00105 \\
\hline NLR & $2.5 \pm 1.1$ & $2.8 \pm 1.3$ & 0.609 \\
\hline \multicolumn{4}{|l|}{$\mathrm{LDH}, \mathrm{IU} / \mathrm{I}$} \\
\hline (Mean \pm SD) & $697.7 \pm 198.8$ & $743.8 \pm 211.4$ & 0.6921 \\
\hline \multicolumn{4}{|l|}{ CRP, mg/l } \\
\hline (Mean \pm SD) & $34.6 \pm 17.6$ & $41.1 \pm 19.8$ & 0.4554 \\
\hline \multicolumn{4}{|l|}{ Pharmacological treatment } \\
\hline Antiviral drugs & $16(34.8 \%)$ & $13(37.1 \%)$ & 1 \\
\hline Hydroxychloroquine & $12(26 \%)$ & $17(48.6 \%)$ & 0.63 \\
\hline Steroids & $39(84.8 \%)$ & $18(51.4 \%)$ & 0.0007 \\
\hline Persistent symptoms on follow-up & $30(65.2 \%)$ & $24(68.5 \%)$ & 0.8148 \\
\hline
\end{tabular}

CT computed tomography, BMI body mass index, NLR neutrophil to lymphocyte ratio, CRP C-reactive protein, $L D H$ lactate dehydrogenase, WBC white blood cell count, COPD chronic obstructive pulmonary disease, CKD chronic kidney disease, CLD chronic liver disease, CAD coronary artery disease 
Table 2 Comparison of initial and follow-up CT between the two follow-up groups

\begin{tabular}{|c|c|c|c|}
\hline Parameter & $\begin{array}{l}\text { Complete radiological resolution } \\
\text { on follow-up CT ( } n=46 ; 56.8 \%)\end{array}$ & $\begin{array}{l}\text { Residual lung findings } \\
\text { on follow-up CT ( } n=35 ; 43.2 \%)\end{array}$ & $p$ value \\
\hline \multicolumn{4}{|l|}{ Laterality of lung involvement on initial CT } \\
\hline Bilateral & $33(71.8 \%)$ & $29(82.8 \%)$ & 0.2962 \\
\hline Unilateral & $13(28.2 \%)$ & $6(17.2 \%)$ & \\
\hline Number of lobes involved on initial CT & $3 \pm 1.3$ & $3.4 \pm 1.4$ & 0.6351 \\
\hline $\begin{array}{l}\text { Percentage of total lung involvement on initial CT (CT severity } \\
\text { score) }\end{array}$ & $8.1 \pm 2.3$ & $12.4 \pm 3.2$ & 0.03843 \\
\hline \multicolumn{4}{|l|}{ Dominant pulmonary opacity on initial $\mathrm{CT}$} \\
\hline Pure GGO & $34(73.9 \%)$ & $24(68.6 \%)$ & 0.6266 \\
\hline Consolidation & $4(8.7 \%)$ & $6(17.1 \%)$ & 0.3155 \\
\hline Mixed pattern & $8(17.4)$ & $5(14.3 \%)$ & 0.7686 \\
\hline \multicolumn{4}{|l|}{ Dominant pulmonary opacity on follow-up CT } \\
\hline GGO & N/A & $16(45.7 \%)$ & N/A \\
\hline Linear/curvilinear parenchymal bands & & $9(25.7 \%)$ & \\
\hline Mixed pattern of GGO and parenchymal bands & & $6(17.2 \%)$ & \\
\hline Interlobular septal thickening & & $4(11.4 \%)$ & \\
\hline Bronchiectasis & & $6(17.2 \%)$ & \\
\hline Time lapse between initial and follow-up CT (Mean days \pm SD) & $101.4 \pm 9.8$ & $99.6 \pm 8.7$ & 0.4737 \\
\hline
\end{tabular}

CT computed tomography, GGO ground glass opacity

radiological resolution (11.2 \pm 4.1 days vs $7.6 \pm 2.3$ days $)$ $(p$ value $=0.00033)$

Among the laboratory parameters initial WBC count was significantly different between the two groups of residual and resolved opacities $\left(7987 \times 10^{3} \pm 2465.8\right.$ vs $\left.5343 \times 10^{3} \pm 1456.4\right)(p$ value $=0.00105)$. However, NLR ratio was not statistically different between the two groups. Initial LDH and CRP levels did not differ significantly between the two follow-up groups of resolved and residual opacities ( $p$ values of 0.455 and 0.69 , respectively).

The percutaneous oxygen saturation was significantly more in patients who subsequently demonstrated complete radiological resolution compared with the group demonstrating residual lung opacities (92.3 \pm $3.2 \%$ vs $88.1 \pm 2.2 \%)(p$ value $=0.0013)$.

With regards to the pharmacological treatment, those receiving steroids (dexamethasone or methylprednisolone) showed complete radiological resolution more frequently $(39 / 46 ; 84.8 \%$ vs $18 / 35 ; 51.4 \%)$ ( $p$ value $=$ 0.0007 ) compared with the group showing persistence of lung opacities. However, hydroxychloroquine and antiviral (remedesevir) administered groups did not show any significant difference with regards to disappearance or persistence of lung opacities $(p$ value $=1$ and 0.63 , respectively).

Patients who demonstrated residual lung opacities on follow-up CT had a significantly higher percentage of

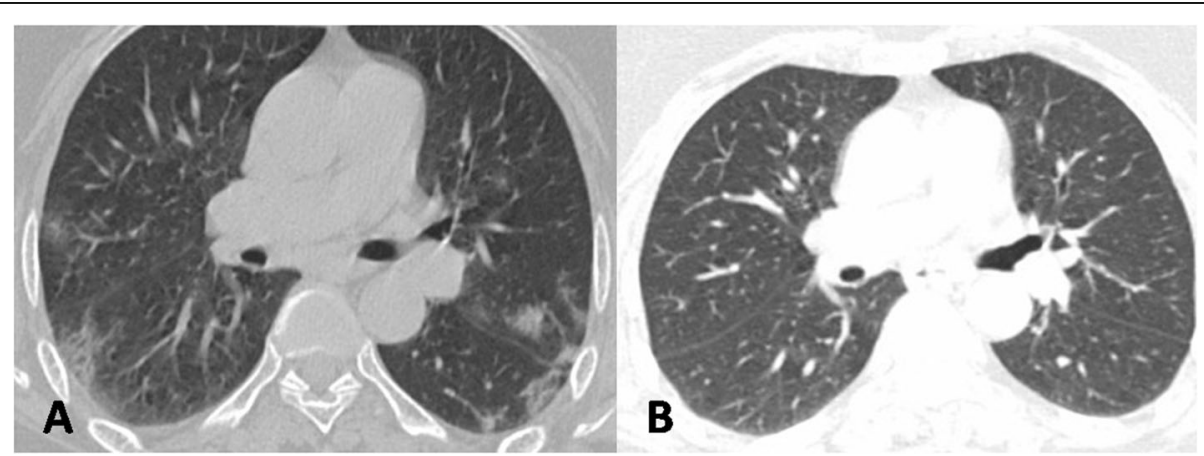

Fig. 1 Axial chest CT image in lung window settings of a 49-year-old COVID-19 patient showing multifocal subpleural ground glass opacities (GGOs) in both lungs at the time of hospital admission (a). Follow-up CT performed 93 days after the first CT reveals complete resolution of GGOs (b) 
total lung involvement (CT severity score $12.4 \pm 3.2$ ) on initial CT compared with the group demonstrating complete radiological resolution (CT severity score $8.1 \pm$ 2.3) $(p$ value $=0.03843)$.

The type of dominant pulmonary opacities on initial CT did not determine the future resolution or persistence of opacities as GGO was the commonest lung opacity observed in $73.9 \%$ of the resolved group and $68.6 \%$ of the residual group ( $p$ value $=0.6266$ )

Similarly, unilateral or bilateral lung involvement or the number of lung lobes involved on initial CT did not differ significantly between the two groups of residual or resolved opacities ( $p$ values $=0.2962$ and 0.6351, respectively).

Only 33.3\% (27/81) patients were completely free of any symptoms on follow-up, while $63.7 \%(54 / 81)$ reported one or more persistent symptoms. The commonest symptoms reported were fatigue $(24 / 54 ; 44.4 \%)$, chest discomfort or pain $(16 / 54 ; 29.6 \%)$, dyspnea $(14 / 54$; $25.9 \%)$ and joint pains $(6 / 54 ; 11.1 \%)$. However, a comparison of persistent symptoms between two follow-up groups of resolved and residual lung findings did not show any statistically significant difference between them (Table 1).

Dominant pulmonary opacities observed on follow-up CT included GGO (16/35; 45.7\%), linear/curvilinear subpleural parenchymal bands (9/35 25.7\%), mixed pattern of GGO and parenchymal bands $(6 / 35 ; 17.2 \%)$, and interlobular septal thickening $(4 / 35 ; 11.4 \%)$ (Figs. 2, 3, and 4). Bronchiectasis was observed in $(6 / 35 ; 17.2 \%)$ (Fig. 2).

\section{Discussion}

As COVID-19 continues its relentless march across the world, follow-up data on survivors of infection has begun to emerge. However, the majority of data available at present focuses on the short-term sequelae of infection and data on medium-term radiological findings are scarce. Persistent clinical symptoms were reported in $87 \%$ post-COVID-19 survivors after a mean follow-up of 2 months in an Italian COVID-19 survivor cohort [14]. Ye et al. [15] reported complete radiological resolution in $37 \%$ of survivors, whereas persistent abnormalities consisting predominantly of subpleural parenchymal bands were observed in $47 \%$ survivors after a mean follow-up of 64 days after symptom onset.

You et al. [16] recorded residual lung abnormalities commonly in post-COVID-19 survivors including GGO in $73 \%$ and pulmonary fibrosis in $26 \%$ after a mean of 40 days after discharge from hospital.

Similarly, in a clinico-radiologic follow-up of a cohort of 55 Chinese patients after recovery, it was found that $63 \%$ had persistent symptoms and $71 \%$ had residual lung opacities including interstitial thickening in $27 \%$ after a mean interval of 3 months [5]. The preliminary prepublication findings from a prospective observational cohort presented in European Respiratory Society (ESR) reported lung abnormalities on CT in $88 \%$ at 6 weeks which dropped to $56 \%$ after 12 weeks [17].

The results of the present study suggest that a substantial proportion of patients show residual lung opacities at medium-term follow-up on CT. The persistent lung opacities are potentially suggestive of permanent lung damage which eventually heals with fibrosis. Viral pneumonias may recover without any residual lung damage or fibrosis. However, there are many viral infections of lungs that have been documented to leave the trail of destruction behind in the form of lung fibrosis. H1N1 pneumonia is occasionally complicated by pulmonary fibrosis [17]. However, the percentage of patients showing residual lung abnormalities post recovery have been reported in $22 \%$ survivors of $\mathrm{H} 7 \mathrm{~N} 9,33 \%$ of MERS, and $38 \%$ of SARS after a mean time interval of $6,2-8$, and 6 months, respectively $[18,19]$. In comparison, $45 \%$ of

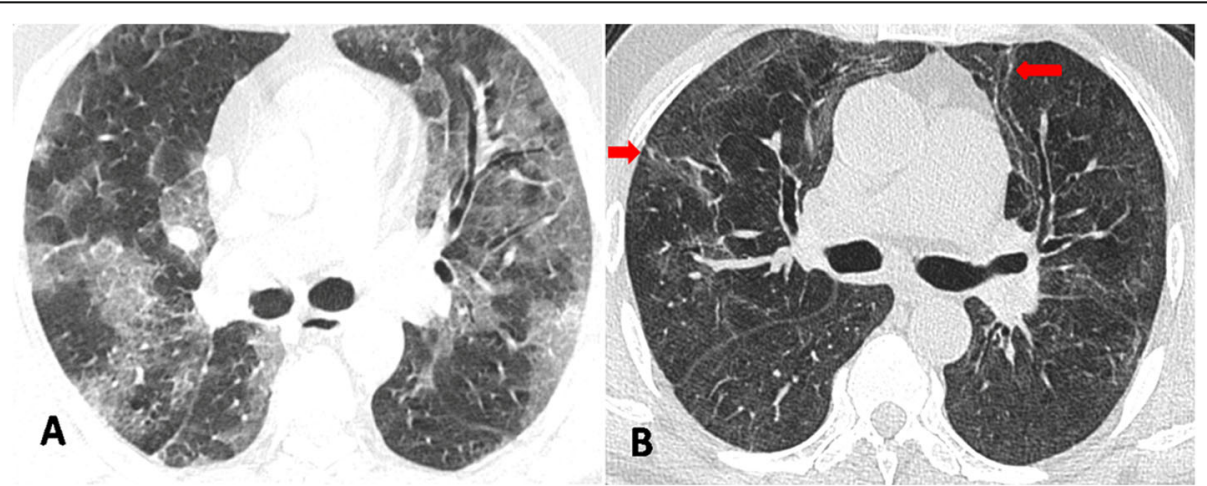

Fig. 2 Axial chest CT image in lung window settings of a 52-year-old COVID-19 patient showing diffuse ground glass opacification in both lungs just below the level of the carina at the time of hospital admission (a). Follow-up CT performed 97 days after the first CT reveals residual faint ground glass opacities in both lungs with architectural distortion and bronchiectasis (red arrows) (b) 


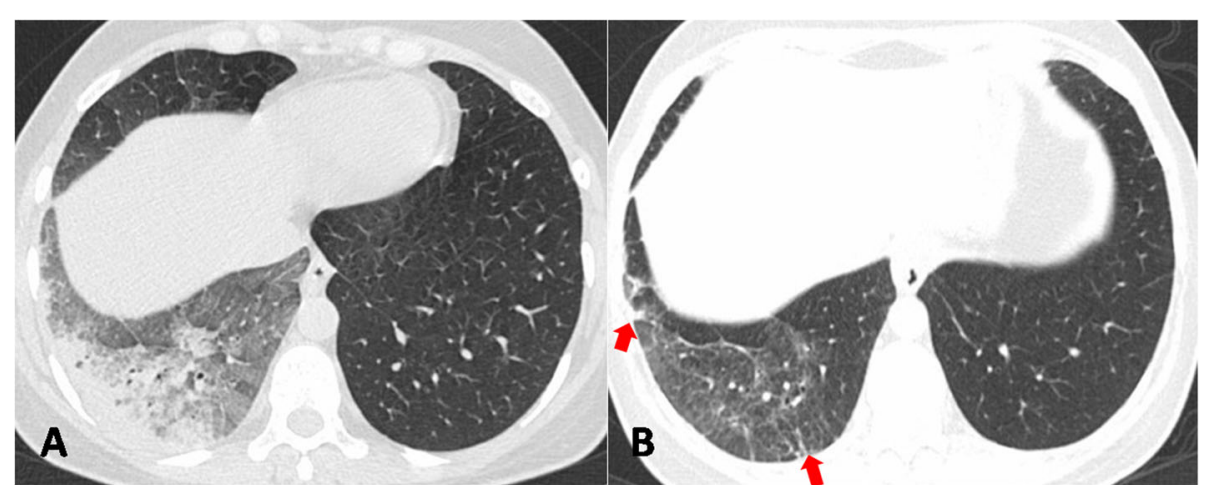

Fig. 3 Axial chest CT image in lung window settings of a 36-year-old COVID-19 patient showing a mixed GGO and consolidation in right lower lobe at admission (a). Follow-up CT performed 92 days later reveals residual GGO with subpleural parenchymal bands (red arrows) (b)

post-COVID-19 survivors showed residual lung abnormalities after a mean follow-up time of more than 3months.

There were many important demographic, clinical, laboratory, and imaging characteristics that were more frequently associated with persistence of residual lung changes. Older age, obesity, preexisting comorbidity, lower oxygen saturation, prolonged hospital stay, ICU admission, and a higher WBC count during acute phase of illness were more commonly associated with residual lung changes on follow-up. Similarly, patients having a more severe lung involvement during initial phase of the disease, as assessed by semi quantitative CT severity score were more likely to have residual lung findings on medium-term follow-up. The more severe the lung injury the greater is the inflammatory response which in turn incites fibroblastic response leading to pulmonary fibrosis [20]. Administration of steroids was associated with lesser probability of having residual lung abnormalities on follow-up which suggests that steroid may have a role in modifying the course of illness and preventing permanent lung damage. A biphasic model of the disease has been proposed to understand the course of COVID19 [21]. While the symptoms and respiratory dysfunction in the first phase are related to viral replication, in the second phase the symptoms are predominantly due to inflammatory response [21]. Anti-inflammatory agents like steroids have been found to improve the outcome in COVID-19 pneumonia by checking the inflammatory response and the same mechanism may hasten the clearance of lung opacities [21-23]. At this stage, however, it is admittedly too early to extrapolate the potential role of steroids in dissipation or resolution of lung opacities in COVID-19 based on the limited data presented in our study. Further prospective studies with large number of patients should be performed to validate the findings of our study.

Though the optimal time for follow-up imaging to assess for radiological clearance is not known, current guidelines of British Thoracic Society recommend a 12week follow-up to provide adequate time for lung abnormalities to resolve which also ensures that non-resolving findings are addressed sufficiently early [19]. A follow-up $\mathrm{CT}$ is not recommended for patients without pneumonia
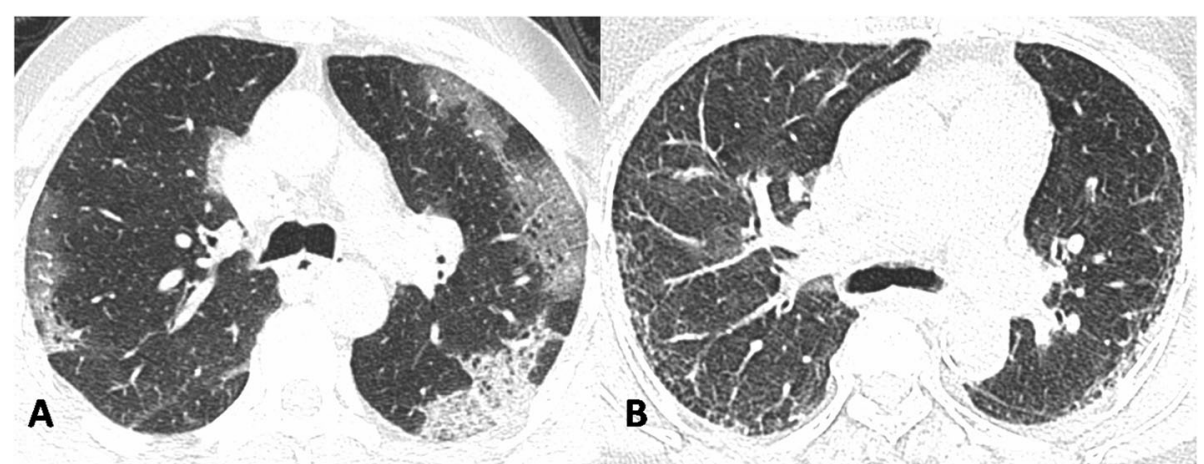

Fig. 4 Axial chest CT image in lung window settings of a 51-year-old COVID-19 patient at the level of carina obtained at admission reveals elongated GGOs in the subpleural lung (a). Follow-up CT performed 107 days later reveals residual faint GGOs with interlobular septal thickening in the subpleural lung (b) 
during the initial active phase of disease nor in patients who show complete resolution of lung findings on follow-up CXR at the time of discharge. High-resolution $\mathrm{CT}$ is indicated for evaluation of patients with persistent significant lung abnormalities on follow-up CXR and in patients with ongoing respiratory symptoms or physiological impairment $[19,24]$.

The medium-term follow-up data is envisaged to provide important information which will guide in devising a structured follow-up plan of COVID-19 survivors to mitigate the long-term sequelae of the disease. Whether the CT findings observed on medium-term follow-up resolve or lead to permanent pulmonary fibrosis will only become apparent with longer-term follow-up.

This study has some limitations. Firstly, only 81 patients were enrolled in the study. A large sample size is desirable to study the pulmonary sequelae of COVID-19. Secondly, we did not perform pulmonary function tests on follow-up which could give more precise information on the physiological impairment of the lungs. Third, we did not perform CT angiography or perfusion imaging to study the burden of residual pulmonary thrombosis. It is being increasingly suggested that pulmonary vascular sequelae may be an important long-term effect of COVID-19 and the residual clot burden can be demonstrated by CT perfusion imaging.

\section{Conclusion}

Nearly half of the COVID-19 survivors had residual lung abnormalities after a time interval of 3 months or more. Older age, obesity, presence of preexisting comorbidity, longer duration of hospital stay, ICU admission, higher initial WBC count, a higher initial CT severity score, and a lower rate of steroid administration are associated with more prevalence of residual lung abnormalities in COVID-19 survivors.

\section{Abbreviations}

COVID-19: Coronavirus disease-19; RT-PCT: Reverse transcriptase polymerase chain reaction; CT: Computed tomography; CXR: Chest X-ray; SARS: Severe acute respiratory syndrome; MERS: Middle East respiratory syndrome

\section{Acknowledgements}

The authors are grateful to the department of radiology and respiratory medicine for their support throughout.

\section{Authors' contributions}

P.A, W.H, S.N, and J.M conceptualized the study and performed or assisted in the collection and analysis of data. P.A and W.H wrote the manuscript which was supervised and edited by S.N and J.M. All authors have read and approved the manuscript and ensure that this is the case.

\section{Funding}

No funding was required for this study.

\section{Ethics approval and consent to participate}

The study was duly approved by the Institutional Ethical Committee (IEC) of Government Medical College, Srinagar, Jammu and Kashmir, India. A reference number was not applicable. The requirement of written patient consent was waived off.

\section{Consent for publication}

Not applicable

\section{Competing interests}

The authors declare no competing interests.

\section{Author details}

'Department of Radiodiagnosis, Sher-i-Kashmir Institute of Medical Sciences, Srinagar, Jammu \& Kashmir, India. ${ }^{2}$ Department of Radiodiagnosis, Government Medical College, Srinagar, Jammu \& Kashmir, India.

${ }^{3}$ Department of Respiratory Medicine, Chest Disease Hospital, Srinagar, Jammu \& Kashmir, India.

Received: 4 December 2020 Accepted: 3 February 2021

Published online: 16 February 2021

\section{References}

1. World Health Organization. WHO coronavirus disease (COVID-19) dashboard. https://covid19.who.int/ Accessed 21 Nov 2020.

2. Revel MP, Parkar AP, Prosch H, Silva M, Sverzellati N, Gleeson F, Brady A (2020) COVID-19 patients and the Radiology department-advice from the European Society of Radiology (ESR) and the European Society of Thoracic Imaging (ESTI). European radiology. 30(9):4903-4909.

3. https:/www.acr.org/Advocacy-and-Economics/ACR-Position-Statements/ Recommendations-for-Chest-Radiography-and-CT-for-Suspected-COVID19Infection. Accessed 30 Oct 2020.

4. Rubin GD, Ryerson CJ, Haramati LB, Sverzellati N, Kanne JP, Raoof S, Schluger NW, Volpi A, Yim JJ, Martin IB, Anderson DJ (2020) The role of chest imaging in patient management during the COVID-19 pandemic: a multinational consensus statement from the Fleischner Society. Chest. 158(1):106-116.

5. Zhao YM, Shang YM, Song WB, Li QQ, Xie H, Xu QF, Jia JL, Li LM, Mao HL, Zhou XM, Luo H (2020) Follow-up study of the pulmonary function and related physiological characteristics of COVID-19 survivors three months after recovery. EClinicalMedicine. 25:100463

6. Hui DS, Joynt GM, Wong KT, Gomersall CD, Li TS, Antonio G, Ko FW, Chan MC, Chan DP, Tong MW, Rainer TH (2005) Impact of severe acute respiratory syndrome (SARS) on pulmonary function, functional capacity and quality of life in a cohort of survivors. Thorax. 60(5):401-409

7. Das KM, Lee EY, Singh R, Enani MA, Al Dossari K, Van Gorkom K, Larsson SG, Langer RD (2017) Follow-up chest radiographic findings in patients with MERS-CoV after recovery. Indian J Radiol Imaging. 27(3):342

8. Parry AH, Wani AH, Shah NN, Yaseen M, Jehangir M (2020) Chest CT features of coronavirus disease-19 (COVID-19) pneumonia: which findings on initial CT can predict an adverse short-term outcome? BJR| Open 2: 20200016

9. Huang Y, Yan Tan C, Wu J, Zhu Chen M, Guo Wang Z, Yun Luo L, Rong Zhou X, Ran Liu X, Ling Huang X, Can Yuan C, Lin Chen C. Impact of coronavirus disease 2019 on pulmonary function in early convalescence phase

10. Li M, Lei P, Zeng B, Li Z, Yu P, Fan B, Wang C, Li Z, Zhou J, Hu S, Liu H (2020) Coronavirus disease (COVID-19): spectrum of CT findings and temporal progression of the disease. Academic radiology. 27(5):603-608.

11. Parry AH, Wani AH, Yaseen M, Shah NN, Dar KA (2020) Clinicoradiological course in coronavirus disease-19 (COVID-19) patients who are asymptomatic at admission. BJR| Open 2:20200033

12. Hansell DM, Bankier AA, MacMahon H, McLoud TC, Muller NL, Remy J (2008) Fleischner Society: glossary of terms for thoracic imaging. Radiology. 246(3): 697-722

13. Pan F, Ye T, Sun P, Gui S, Liang B, Li L, Zheng D, Wang J, Hesketh RL, Yang $L$, Zheng $C$ (2020) Time course of lung changes at chest $C T$ during recovery from coronavirus disease 2019 (COVID-19). Radiology. 295(3):715-721.

14. Carfi A, Bernabei R, Landi F (2020) Persistent symptoms in patients after acute COVID-19. JAMA. 324(6):603-605 
15. Ye T, Fan Y, Liu J, Yang C, Huang S, Xiong B (2020) Follow-up chest CT findings from discharged patients with severe COVID-19: an 83-day observational study. Nuclear Med Med Imaging:1-15

16. You J, Zhang $L$ (2020) Abnormal pulmonary function and residual $C T$ abnormalities in rehabilitating COVID-19 patients after discharge: a prospective cohort study. J Infect.

17. Udwadia ZF, Koul PA, Richeldi L. Post-COVID lung fibrosis: the tsunami that will follow the earthquake. Lung India [Epub ahead of print]. Available from: https://www.lungindia.com/preprintarticle.asp?id=300649. [cited 2020 Dec 30]

18. Wang $Q$, Jiang H, Xie Y, Zhang T, Liu S, Wu S, Sun Q, Song S, Wang W, Deng X, Ren L (2020) Long-term clinical prognosis of human infections with avian influenza A (H7N9) viruses in China after hospitalization. EClinicalMedicine. 20:100282.

19. George PM, Barratt SL, Condliffe R, Desai SR, Devaraj A, Forrest I, Gibbons MA, Hart N, Jenkins RG, McAuley DF, Patel BV (2020) Respiratory follow-up of patients with COVID-19 pneumonia. Thorax. 75(11):1009-1016.

20. Wallace WA, Fitch PM, Simpson AJ, Howie SE (2007) Inflammationassociated remodelling and fibrosis in the lung-a process and an end point. Int J Exp Pathol. 88(2):103-110

21. Mikulska M, Nicolini LA, Signori A, Di Biagio A, Sepulcri C, Russo C, Dettori S, Berruti M, Sormani MP, Giacobbe DR, Vena A (2020) Tocilizumab and steroid treatment in patients with COVID-19 pneumonia. Plos one. 15(8):e0237831

22. Sanders JM, Monogue ML, Jodlowski TZ, Cutrell JB (2020) Pharmacologic treatments for coronavirus disease 2019 (COVID-19): a review. JAMA. 323(18):1824-1836

23. Parry AH, Wani HA, Choh NA, Shah NN, Jehangir M (2021) Spectrum of chest CT manifestations of coronavirus disease (COVID-19): a pictorial essay. Indian J Radiol Imaging. 31(5):170

24. Raghu G, Wilson KC (2020) COVID-19 interstitial pneumonia: monitoring the clinical course in survivors. Lancet Respir Med. 8(9):839-842

\section{Publisher's Note}

Springer Nature remains neutral with regard to jurisdictional claims in published maps and institutional affiliations.

\section{Submit your manuscript to a SpringerOpen ${ }^{\circ}$ journal and benefit from:}

- Convenient online submission

- Rigorous peer review

- Open access: articles freely available online

- High visibility within the field

- Retaining the copyright to your article 